\title{
Application of transient pulses on power supply cables by using commercial of the shelf components
}

\author{
J. Hagmann ${ }^{1}$, L.-O. Fichte ${ }^{1}$, S. Dickmann ${ }^{1}$, M. Schaarschmidt ${ }^{2}$, and S. Potthast ${ }^{2}$ \\ ${ }^{1}$ Helmut-Schmidt-Universität/Universität der Bundeswehr Hamburg, Hamburg, Germany \\ ${ }^{2}$ Wehrwissenschaftliches Institut für Schutztechnologien - ABC-Schutz (WIS), Munster, Germany
}

\begin{abstract}
This paper deals with the development of ultra wide band pulse devices using merchantable hardware. The major goal is to create wide band transients and pulses with simple electronic equipment and to achieve high voltages and low pulse rise times. The propagation of these signals on common power lines are examined to analyse and forecast the effects on attached devices.
\end{abstract}

\section{Introduction}

The basic idea of this research project is the application of pulses on power lines using "commercial of the shelf" components (COTS). Therefore it is necessary to generate wide band transient pulses which can propagate along a power line system and produce cable bounded effects with the attached devices. One of the main elements for this research is a so called Taser (Fig. 1) or stun-gun (Fig. 2), which are used as a high voltage power supply for the forthcoming tests.

The main analysis deals with the disturbing effects of these kinds of power supplies on attached components, e.g. computers and all kinds of electronic devices.

Conducted emission and cable coupling are the main couple mechanisms for this chosen problem. The high voltage transients or disturbances are the source for the IEMI. The power supply cables and data communication cables are used to transfer the transients from the source to the receptor.

\section{Stun-gun}

This research starts with the analysis of different simple high voltage sources, which are used to generate a high voltage out of simple $9 \mathrm{~V}$ batteries. These high voltage sources should

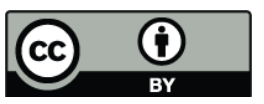

Correspondence to: J. Hagmann (johannes.hagmann@hsu-hh.de)

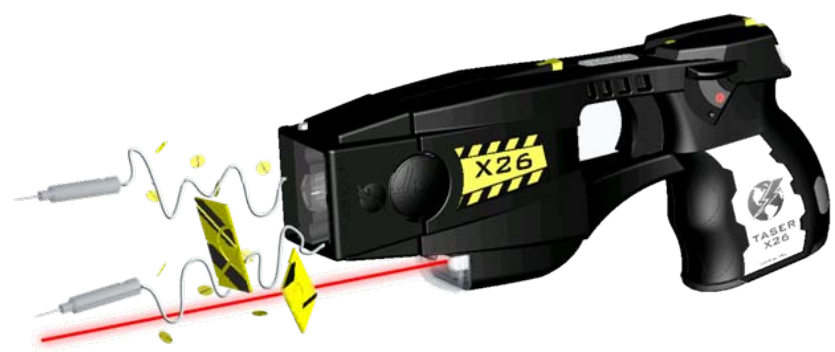

Fig. 1. Taser.

be handy to be mobile in a small housing. Therefore, some typical mobile voltage sources were analysed, like stun-guns, tesla-transformers and fence-energizers. The following properties should be complied by the ideal source:

- high output voltage,

- network-independent power supply,

- compact casing box.

Because of the potential high output voltage of ca. $250 \mathrm{kV}$ and the compact and lightweight housing a stun-gun, a kind of the electroshock devices, was chosen. The mechanism of action for these devices is based on the well known Taser (Fig. 1). This stun gun fulfils the made demands above. The figure (Fig. 2) shows the components of the examined stungun: battery case (9V-Block), dc-ac converter, intermediate circuit with transformer, tesla-stage with spark gap, teslatransformer, contact electrodes. The stun-gun is easily to obtain on the legal market and they are easily to handle. Older investigations have shown, that these devices have not only been used for the planned application but have also been used for IEMI, e.g. to influence alarm systems and computers by direct contact.

The working mechanism of a stun-gun is shown in the schematic (Fig. 3). The gun is supplied by a

Published by Copernicus Publications on behalf of the URSI Landesausschuss in der Bundesrepublik Deutschland e.V. 

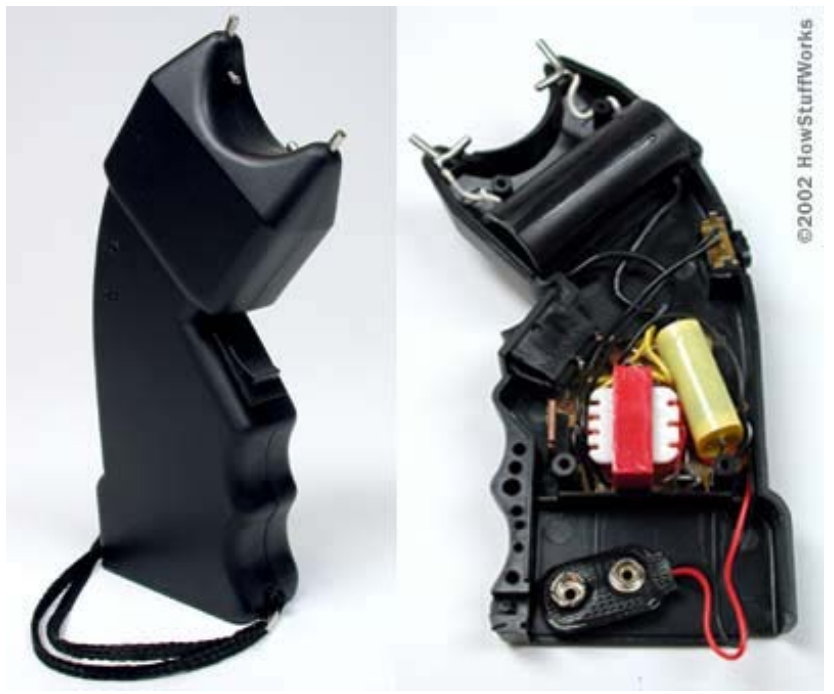

Fig. 2. Stun-Gun.

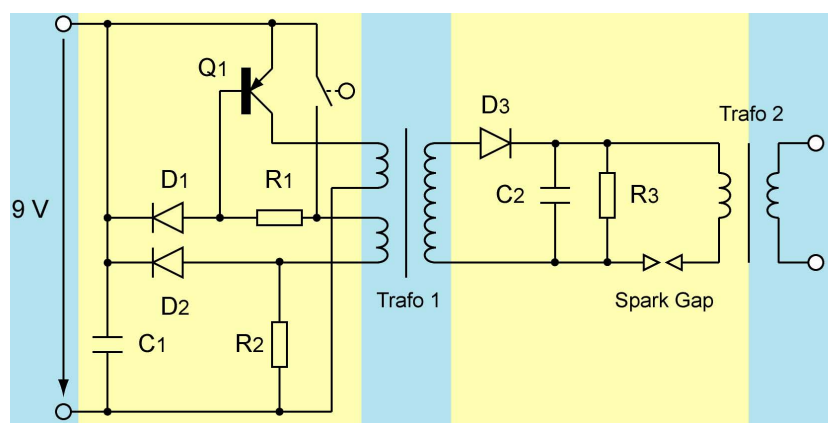

Fig. 3. Schematic of a stun-gun.

9V-block-battery. This dc voltage is converted into an ac voltage by a oscillator circuit. This ac voltage is transformed up to ca. $1.5 \mathrm{kV}$. The second stage is characterized by a tesla transformer. The diode $D_{3}$ is used to commutate the high ac voltage and loads the capacitor $C_{2}$. The spark gap fires, when the breakthrough voltage of about $1.5 \mathrm{kV}$ is reached. While the spark gap fires, the voltage at the electrodes is theoretically transformed up to the range of $100-250 \mathrm{kV}$, before inner sparks could damage the transformer or internal electronics in the stun gun.

\section{Using the stun-gun as pulse source}

For the actual application of transient pulses on the power supply cables the stun-gun can be used directly. Therefore the contact electrodes are connected to the power lines and the stun gun pulse is applied on the lines. The effects of disturbances has to be analysed.

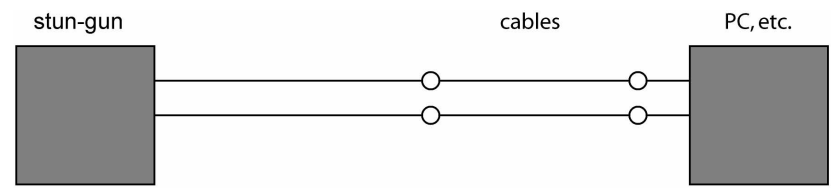

Fig. 4. Stun-gun, directly attached.

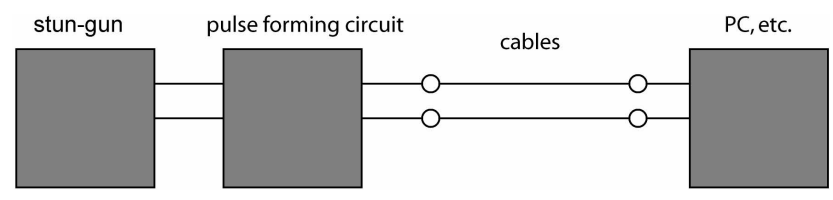

Fig. 5. With pulse forming circuit.

This arrangement (Fig. 4) has some obvious advantages:

- hardly any additional hardware is used,

- the transients are easily to apply,

- high voltage transients can be generated,

- the system is compact and mobile.

The stun gun is commonly designed to interact with biological tissue. Therefore a current (...) is driven through the human body to immobilize a possible attacker. The shape of the pulse is formed to be optimized for the nervous system. The pulses are bipolar and have only a narrow bandwidth in the lower frequency range. For the planned applications as a UWB-pulse-source in the upper frequency range the stungun is not useful directly. As an alternative for the direct use of the stun gun a pulse forming network was considered (Fig. 5. This pulse forming stage is loaded by the stun-gun. The stun-gun is used as a current source, which can provide a high voltage. The pulse forming stage is designed to generate a double exponential transient or a damped sinus. Therefore a high amplitude for the transient and a short rise time of the pulse and high frequency range are needed and taken into account.

\section{Pulse forming network}

The schematic of the used pulse forming circuit is shown in (Fig. 6). A capacitor $C$ is used to store the electric charge. It is charged by a high voltage source via the diode $D$, with a high breakdown voltage $15 \mathrm{kV}$. The spark gap should fire at a voltage of some $10 \mathrm{kV}$ and can be interpreted as a bypass, while firing. The designated voltage for the spark gap can be set by the distance of the electrodes. The pulse is applied on a line, while the capacitor is discharged via a parasitic inductance to the applied line. 


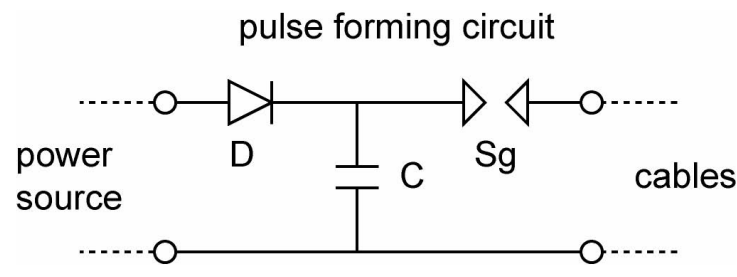

Fig. 6. Pulse forming network.

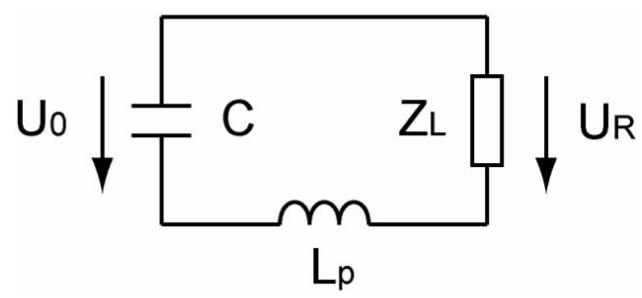

Fig. 7. Parasitic elements.

The pulse forming network should be useful for wide frequency band. Therefore it is useful to match the pulse forming network to the line impedance of the attached cables. The circuit has to be compact, due to the parasitic effects of the circuit. Especially the current loop and it's spanned area of the capacitor-sparkgap-circuit form an inductance (Fig. 6), which increases the rise time of the pulse and decreases the frequency range. In the following analysis the line impedance of the lines, which are assumed to be ohmic, are expressed by the resistance $R$ (Fig. 7). The parasitic inductance, which is described by the area spanned by the flowing current, is described by $L_{p}$. Which voltage $U_{\mathrm{R}}$ can be applied on the line and how can the voltage be calculated, according to the charge voltage at the capacitor $U_{0}$. Using the Laplace's transformation, the solution for this problem can be derieved. The solution starts with:

$U_{\mathrm{R}}(s)=U_{0} \cdot \frac{R}{L} \cdot \frac{1}{s^{2}+s \frac{R}{L}+\frac{1}{C L}}$.

For the transformation of this equation (Eq. 1) from the frequency domain back into the time domain the correspondence tables are used via a substitution:

$U_{\mathrm{R}}(s)=U_{0} \cdot \frac{R}{L} \cdot \frac{1}{\left(s-s_{1}\right)\left(s-s_{2}\right)}$.

With the according Values:

$s_{1,2}=-\frac{R}{2 L} \pm \sqrt{\frac{R^{2}}{4 L^{2}}-\frac{1}{C L}}$.

As a solution in the time domain can be dereived by the transformation:

$U_{\mathrm{R}}=U_{0} \cdot \frac{R}{L} \cdot \frac{1}{\sqrt{\frac{R^{2}}{4 L^{2}}-\frac{1}{C L}}} \cdot \mathrm{e}^{-\frac{R}{2 L} t} \cdot\left[\sinh \left(\sqrt{\frac{R^{2}}{4 L^{2}}-\frac{1}{C L}} \cdot t\right)\right]$.

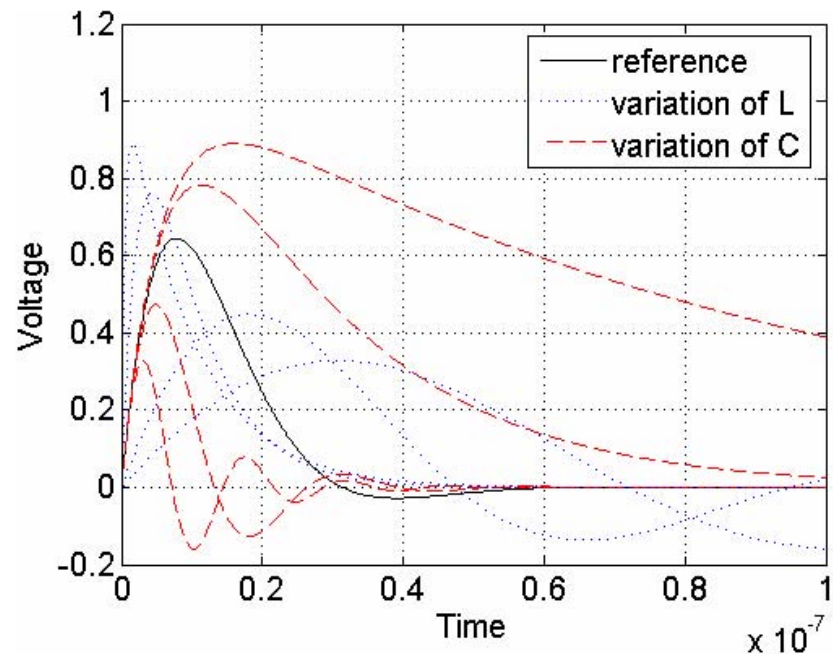

Fig. 8. Double-exponential pulse.

In Eq. (4) the common solution for the voltage $U_{\mathrm{R}}$ on the start point of the lines is given. In this equation an exponentiell damping factor and a sinh-function are given with a prefactor according to the voltage amplitude. These combined terms generate a double-exponential function, which can be transformed into a damped sinus according to the circuit elements $L, C, R$ (Fig. 8). It can be seen, that the increase of the inductance $L$ and the decrease of the capacitor $C$ produce a damped sinus.

The expected voltage shape for $U_{\mathrm{R}}$ is a double-exponential discharge and the case for the critical damping. Therefore the squareroot term has to be

$\sqrt{\frac{R^{2}}{4 L^{2}}-\frac{1}{C L}} \rightarrow 0$.

Therefore the following inequality is used to determine the case:

$\frac{R^{2}}{4} \geq \frac{L}{C}$

To achieve high frequency spectral parts, it is necessary to increase the values for $s_{1}$ and $s_{2}$. Therefore the parasitic inductance of the circuit has to be very small. The other elements have to be balanced to to fulfil the Eqs. (5) and (6). Especially the parasitic inductance $L_{\mathrm{p}}$ and the capacitor $C$ have to be balanced.

\section{Design}

The main efforts at the design of the pulsforming circuit are: compact design and housing, low parasitic inductance, low peeking-capacitor, high voltage capability of all elements 


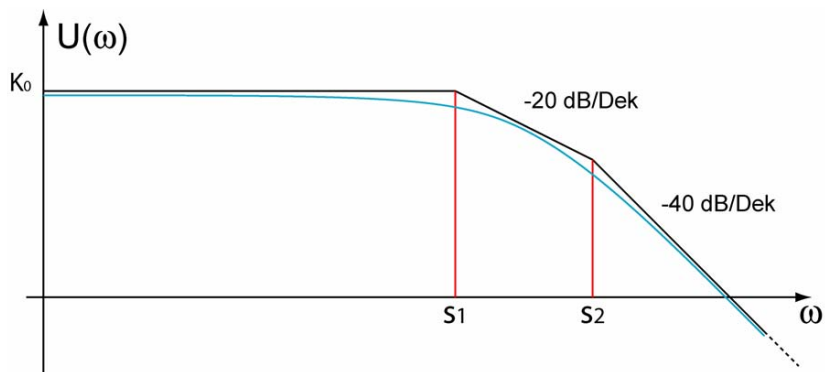

Fig. 9. Pulse in frequency domain.

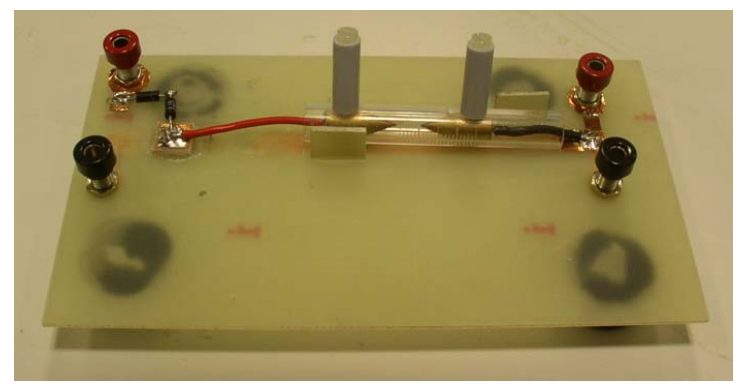

Fig. 10. Simle pulse forming circuit.

and the PCB layers, matching the line impedance of the cables to be attached. The Fig. 10 shows a simple pulse forming network of the first gerneration. The Elements used for this circuit can be seen. These elements include a diode, capacitor and a spark gap together with some contact clamps for voltage supply and for the cables to be attached.

It is possible to generate double-exponential transient pulses, which can propagate on the lines to a destinated receptor. The pulsforming stage is charged onto a voltage of ca. $5 \mathrm{kV}$ and the spark gap fires. The Fig. 11 shows the measured transient on the line. This measured pulse shows a voltage amplitude of $4.9 \mathrm{kV}$ and a rise time of $4.5 \mathrm{~ns}$. During the measurements the capacitive probe $\mathrm{P} 6015$ was used. This probe can only resolute a minimum rise time of $4.5 \mathrm{~ns}$. Therefore the transient's rise time can be lower than the measured.

\section{Transient on lines}

The next analysis deals with the influence of the cables on the pulses and according to the quality of the lines with the attenuation and dispersion along the lines.

In principle, the following tasks have to be analysed:

- breakthrough voltage

- attenuation

- dispersion

- line impedance

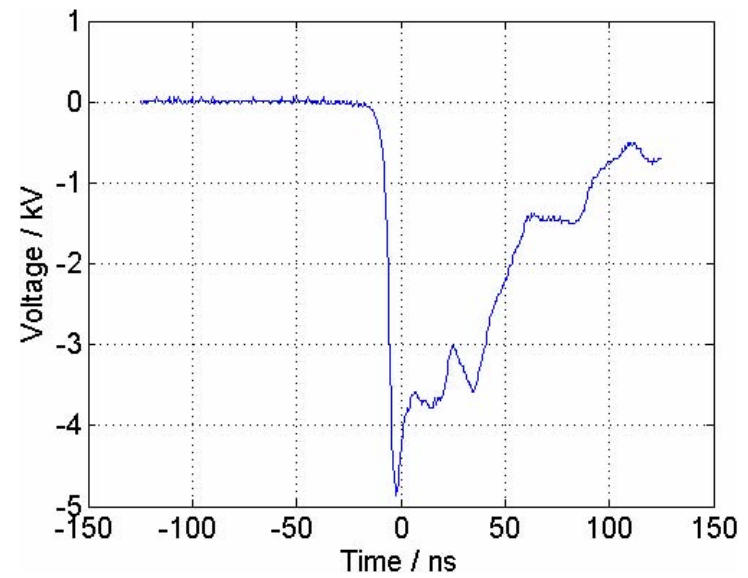

Fig. 11. Measured transient.

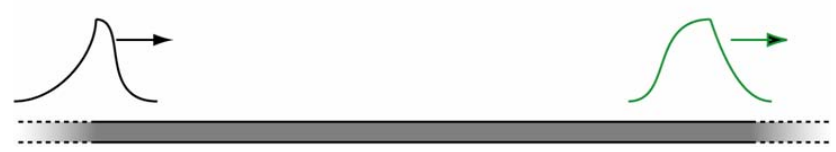

Fig. 12. Pulse on the line.

To determine the influence of the cables on the pulses, NYM $\left(1.5 \mathrm{~mm}^{2}\right)$ power supply cables were used, which are commonly used as power supply cables in the civil households. The line impedance of these lines are ca. $Z_{\mathrm{L}}=82 \Omega$ for flexible cable and $Z_{\mathrm{L}}=75 \Omega$ for semi rigid cables. To determine the attenuation for the cables measurements were done (Fig. 13). The measured values depend on the measured scatter-parameters of the line and show the reflection parameter $S_{11}$ and the transmission parameter $S_{12}$, which represents the attenuation of the CW-signal.

The analysis shows, that the used test cable of $1 \mathrm{~m}$ length provides a linear damping factor depending on the frequency. At the frequency of $1 \mathrm{GHz}$ a damping factor of $8 \mathrm{~dB}$ can be measured, while at a frequency of $200 \mathrm{MHz}$ an attenuation of $2 \mathrm{~dB}$ can be determined. This lines are principally applicable up to a frequency of about $500 \mathrm{MHz}$, because the most relevant cable conducted effects in the attached devices are determined in the frequency range below $f_{0}=300 \mathrm{MHz}$. Assuming linear behaviour of the line system, we can determine dispersion on the line system due to the propagation velocity, which is frequency dependent. This dispersion effect influences the shape of the transient pulse along the line. The rise time $t_{\mathrm{A}}$ of the pulse increases. To determine the nonlinear behaviour of the lines, pulses with different frequency ranges and amplitudes were applied on the power lines. The pulses at the front and back side of the $10 \mathrm{~m}$-line have the same shape, but differences in running time of ca. 50ns (Fig. 14). 

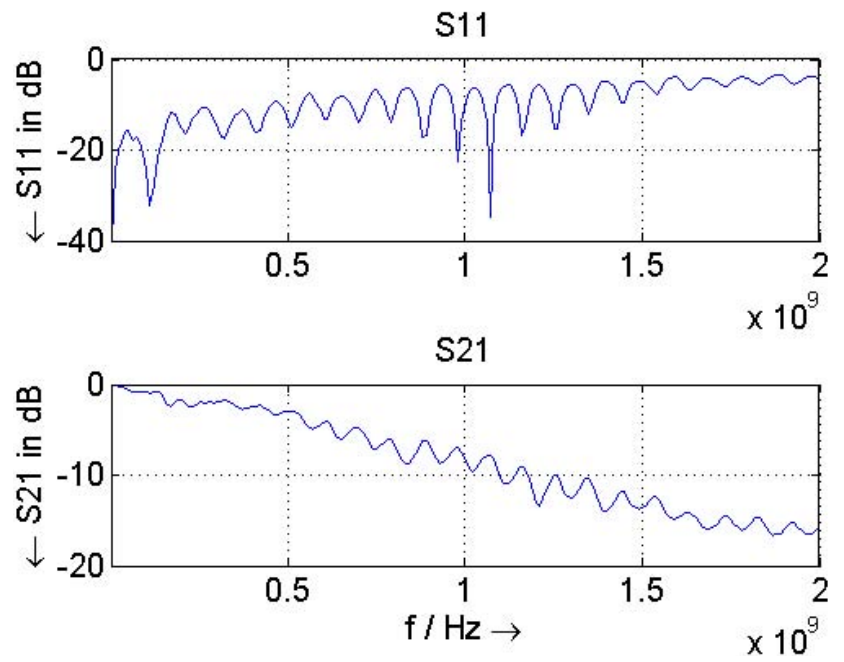

Fig. 13. S-Parameters of a NYM-cable (1m).
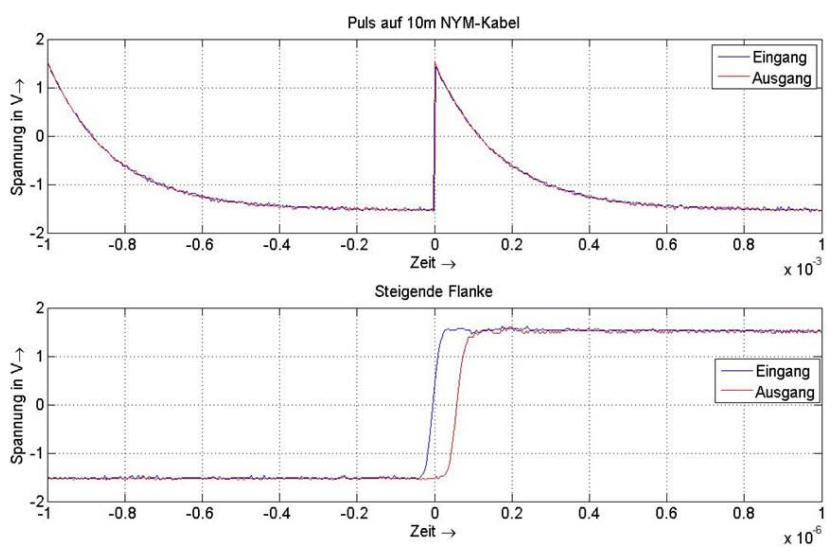

Fig. 14. Pulse propagation on the lines.

\section{Filtering}

Common netfilters in personal computers and electronic devices are designed to filter surges and harmonics near the net frequency $f=0.1-2 \mathrm{kHz}$ from the supply voltage. Another goal of this research is to generate pulses with spectral parts in frequnecy ranges, which are not filtered by the net filters to pass over to the sensitive stages of a device. The next picture shows the filter quality $S_{12}$ of a common personal computer.

The filter shows different behaviour under a common mode and a differential mode source. Some gaps in the filtering ability of the filter can be seen (Figs. 15, 16). Using a differential mode signal a gap in the filtering ability is shown at about 200MHz. The attenuation effect of the filter is low in the frequency range of $1-2 \mathrm{GHz}$ (Fig. 15). Using a common mode some other frequency ranges below $1 \mathrm{GHz}$ with a low attenuation below $10 \mathrm{~dB}$ can be found (Fig. 16). These analysis of the filter attenuation depends on CW signals and steady state vibrations of the filters. To include the transient

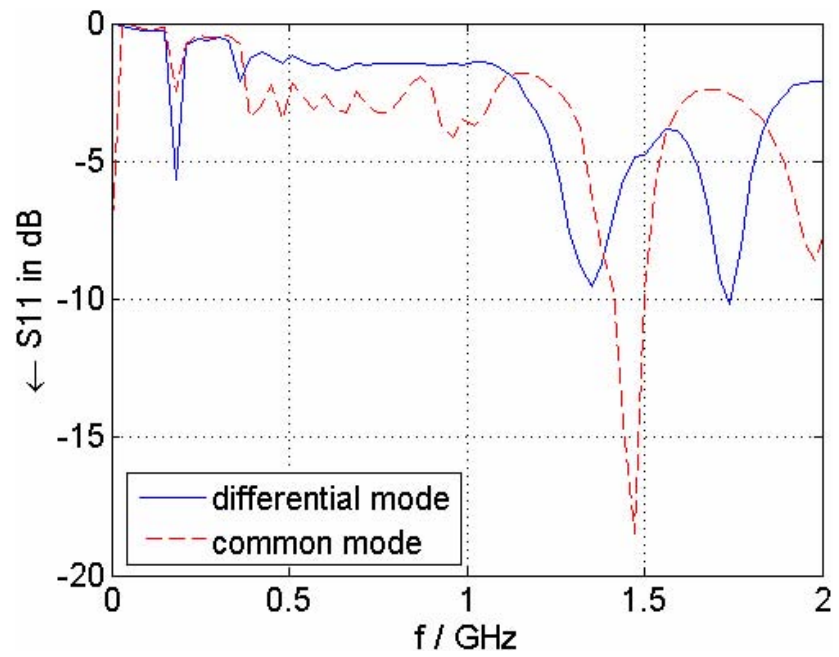

Fig. 15. Filter quality, measured between phase and ground.

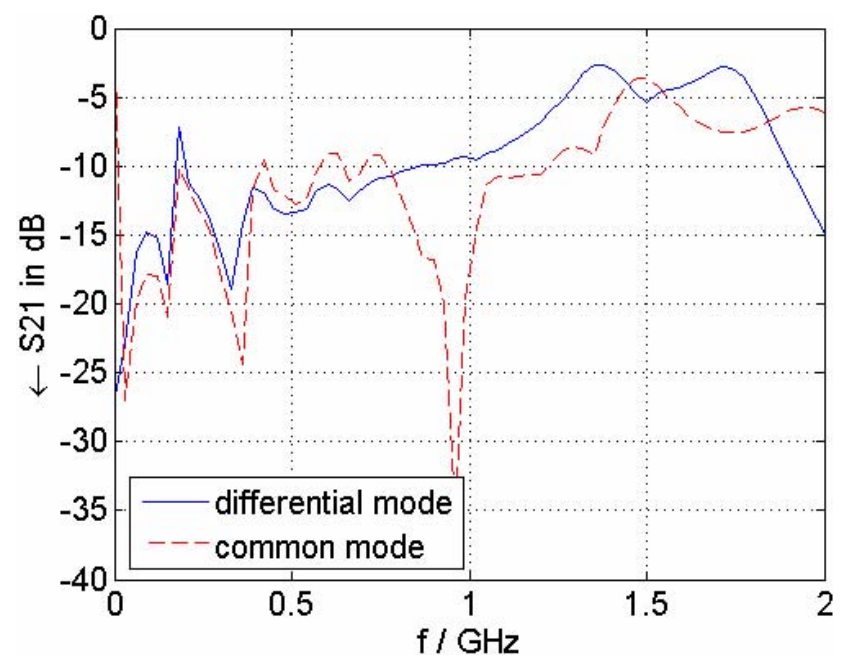

Fig. 16. Filter quality, measured between phase and ground.

oscillation of the capacities of the filters, it is necessary to analyse the filters in the time domain. The transient pulses can be that fast, that the filters are not able to react. This is a field for further research.

\section{Conclusions}

In this paper some steps to produce a simple pulse generator were shown. The generated pulses have a high voltage and low puse rise times. Therefore the pulses have a wide frequency bandwidth. For the application in the case of IEMI, some power supply cables were attached and filters were analysed to determine the propagation behaviour of the transients on these lines. 


\section{References}

Benford, J., Swegle, J. A., and Schamiloglu, W.: High Power Microwaves, Second Edition, Taylor \& Friends, New York, 2007.

Hilberg, W.: Impulse auf Leitungen, Oldenbourg, Mnchen, 1981.

Hölzler, E. and Holzwarth, H.: Pulstechnik, Band 1, Springer, Berlin, 1982.

Hölzler, E. and Holzwarth, H.: Pulstechnik, Band 2, Springer, Berlin, 1984.

Mesyats, G. A.: Pulsed Power, Springer, Netherlands, 2004.
Paul, C. R.: Analysis of Multiconductor Transmission Lines, Wiley Series in Microwave and Optical Engineering, John Wiley \& Sons, 1994.

Paul, C. R.: Introduction to Electromagnetic Compatibility, Wiley Series in Microwave and Optical Engineering, John Wiley \& Sons, 2006.

Schildt, G. H.: Impulstechnik: Grundlagen und Anwendungen, Lyk Informationstechnik, 2008.

Zinke, O. and Brunswig, H.: Hochfrequenztechnik I: Hochfrequenzfilter, Leitungen, Antennen, Springer, Berlin, 1999. 